\title{
Transport and adsorption of nano-colloids in porous media observed by Magnetic Resonance Imaging
}

\author{
A.P. Lehoux ${ }^{a, b, c}$, P. Faure ${ }^{a}$, E. Michel ${ }^{b}$, D. Courtier-Murias ${ }^{a}$, S. Rodts ${ }^{a}$, P. Coussot ${ }^{a}$ \\ ${ }^{a}$ Université Paris-Est, Laboratoire Navier (ENPC, IFSTTAR, CNRS), Champs-sur-Marne, France \\ ${ }^{\mathrm{b}}$ EMMAH, INRA, Université d'Avignon et des Pays de Vaucluse, Avignon, France \\ ${ }^{\mathrm{C}}$ Present address: Department of Earth Sciences, Uppsala University, Villavägen 16, Uppsala 75236, \\ Sweden
}

\begin{abstract}
We use Magnetic Resonance Imaging (MRI) to follow the adsorption of colloids during their transport through a porous medium (grain packing). We injected successive pulses of a suspension of nano-particles able to adsorb onto the grains. To get quantitative information we carry out 2D imaging and 1D measurements of the evolution in time of the distribution profile of all particles (suspended or adsorbed) in cross-sectional layers along the sample axis during the flow. For the first injections we observe the 1D profile amplitude progressively damping as particles advance through the sample, due to their adsorption. 2D imaging shows that successive injections finally results in a coverage of grains by adsorbed particles regularly progressing along the sample. The analysis of the results makes it possible to get a clear description of the adsorption process. In our specific case (particle charged oppositely to the adsorption sites) it appears that the particles rapidly explore the pores and adsorb as soon as they encounter available sites on grains, and the surplus of particles go on advancing in the sample. A further analysis of the profiles makes it possible to distinguish the respective concentration distribution of suspended and adsorbed particles over time at each step of the process.
\end{abstract}

\section{Introduction}

The transport and retention of colloids in soil is of great environmental concern for two reasons. First, colloids can be pollutants such as viruses, bacteria, and nanoparticles. Industrial and agricultural activities may lead to the leaching of contaminants through the soil via direct leaking from unintentional release, reuse of wastewater, landfills and agricultural use of products containing artificial nanomaterials (Nowach and Bucheli 2007). These contaminants in soil can be harmful for plants and microbial communities as they might reach the groundwater and cause pollutions. Secondly, the transport of autochthonous colloids in soils (e.g. clays, organic matter, iron oxides, and other minerals) can be a vector of low-solubility contaminants such as radionuclides and trace metals, because of their similar chemical surface properties (McCarthy and Zachara 1989, Ryan and Elimelech 1996).

Many studies aimed at understanding the mechanisms of colloidal transport and adsorption, which may involve complex interactions between colloids, soil matrix, pore water and air, with the 
ambition to predict where and when soil and water pollution will occur (Majdalani et al. 2007; Cey et al. 2009; Diaz et al. 2010). One of the challenges with soils is to identify the relative importance of the different possible effects (transport, adsorption, dispersion, resuspension, etc) induced by the complex soil's chemical properties, and the impact of the different elements of its structure. In that aim various studies used model porous media such as sand or glass beads as model for soils (Baumann and Werth 2005; Bradford et al. 2007; Bradford et al. 2002; Lakshmanan et al. 2015a; Lakshmanan et al. 2015b; Lehoux et al. 2016; Ramanan et al. 2012; Tufenkji et al. 2005), which allowed studying different physical phenomena separately.

In most cases, these approaches rely on the interpretations of effluent concentration curves (particle breakthrough curves) coming from columns of saturated porous media (Yao et al. 1971; Song and Elimelech 1993; Hahn et al. 2004; Simunek et al. 2006; Diaz et al. 2010). However such approaches provide only the final result of a complex phenomenon developing throughout the sample so that the full validation of a model is a difficult task. Moreover, from MRI (Magnetic Resonance Imaging) of $D_{2} O$ injection in chromatography gel columns a significant band broadening due to column inlet was observed (Harding and Baumann, 2001), and from an analysis of NMR data, it was suggested that imperfect flow injection could also have a significant impact on the dispersion observed from breakthrough curve experiments (Scheven et al., 2007). It was confirmed recently (Lehoux et al. 2016) that entrance and exit effects strongly affect breakthrough curves and that the effective dispersion (i.e. the dispersion determined from direct measurements inside the sample) is much lower than usually assumed, which further illustrates the difficulty to interpret relevantly global information from particle breakthrough curves. In this context it appears crucial to be able to get data from inside the sample, and observe the transport and adsorption processes independently of edge effects.

Lately, various non-invasive visualization techniques have been developed in order to obtain a direct access to the mechanisms occurring in the porous media. In particular ${ }^{1} \mathrm{H}$ Magnetic Resonance Imaging (MRI) of water in saturated samples proved to be efficient to follow particle concentration along a mesoscopic sample. With this technique one records the signal emitted by protons spins after their excitation and during their relaxation back towards equilibrium (with two relaxation times, i.e. $T_{1}$ and $T_{2}$, associated with respectively longitudinal and transverse relaxation of the NMR signal, and which depend on different processes). Baumann and Werth (2005) obtained $T_{1}$ weighted images (density field with signal enhanced for large relaxation time $T_{1}$ ) from which they extracted the distribution of particle concentration in time, converting all MRI signal in concentration of suspended particles, despite an important adsorption. Ramanan et al. (2012), Lakshmanan et al. (2015a), and Lakshmanan et al. (2015b) obtained 2D $T_{2}$-weighted images (same as $T_{1}$-weighted images but with the other relaxation process) of the liquid in coarse grain packings with an excellent resolution allowing to observe the transport in the structure at the pore scale, and deduced detailed characteristics of the evolution of particle distribution in time along the sample. Finally Lehoux et al. (2016) measured directly particle concentration profiles along the sample axis. However in these approaches only concentration profiles of suspended particles are measured and concentration profiles of adsorbed particles have never been reported. Actually it should be expected that adsorbed particles do not contribute to MRI signal in the same way as suspended particles. More precisely, suspended paramagnetic particles are expected to influence the NMR (Nuclear Magnetic Resonance) relaxation rate of water in the bulk phase, whereas adsorbed particles modify the 
relaxation rate of liquid along the pore surface. As a consequence, the effect of particles on water signal depends on their state. In the perspective of a better understanding of transport and adsorption of colloidal particles there is a need for straightforward measurements of the distribution of all particles during their transport in porous media, including adsorbed and suspended particles.

Our work shows the possibility to get more complete information on the transport and adsorption processes by measuring by NMR the evolution in time of the distribution profile of all particles (suspended or adsorbed) in cross-sectional layers along the sample axis. A further analysis of these profiles makes it possible to distinguish the respective concentrations of particles in each state. Under our particular conditions we were able to deduce that the particles get adsorbed almost immediately when they reach a region where adsorption sites are available. The materials and procedure are presented in Section 2. The results are presented and discussed in Section 3.

\section{Material \& methods:}

\section{Porous material}

As a porous medium we used Fontainebleau sand sieved at 200-250 $\mu \mathrm{m}$, washed with concentrated nitric acid (65\%) in a bain-marie for 2 hours, then with a $0.1 \mathrm{M} \mathrm{NaOH}$ solution and finally cleaned with deionized water. Under these conditions the sand grain surface is negatively charged (zeta potential of $-42.11 \mathrm{mV}$ according to Jacobs et al. (2007)). Besides a picture of the grain surface may be found in Jacobs et al. (2007).

The sand was then packed in three columns namely A, B and C ( $15 \mathrm{~cm}$ height, $5 \mathrm{~cm}$ diameter) with deionized water (conductivity of $18.2 \mathrm{M} \Omega . \mathrm{cm}$, referred to as pure water in the following). The columns were built in PMMA (Poly(methyl methacrylate) for minimizing their visibility on MRI data. The columns were wet packed, and the sand was manually stirred regularly during packing to avoid the presence of air bubbles before closing the columns. The total liquid volume in the saturated columns was $114.5+/-1.5 \mathrm{ml}$. The packing porosity deduced from the ratio of this water volume to the column volume was $\varepsilon \approx 0.39$. A paper filter, $420 \mu \mathrm{m}$ thick with a pore size of $30 \mu \mathrm{m}$, was set up at each edge of the columns to prevent the sand from leaking out. We have no particular information on the bead packing characteristics, we just assume that it is rather homogeneous. This is supported by the rather similar progression of the fluid at different radial positions in the column (see Figure 4) ; the unevenness of the front being essentially formed (and fixed) at the entrance; if the packing was significantly heterogeneous it would affect the local permeability and consequently the local velocity would evolve differently at different radial distances.

\section{Colloidal particles}

In the present study we used the well-documented situation of the injection of a suspension of particles in pure water with an opposite charge to that of the porous medium (Elimelech and Song 1992), which are likely to produce an almost instantaneous and irreversible adsorption at the surface of sand grains. As colloidal particles we used superparamagnetic commercially available particles (Molday ION C6Amine) which have an iron oxide core and are covered with surface amino groups. Their iron concentration is $1.49 \times 10^{-20}$ moles of iron per particle and their diameter is $35 \mathrm{~nm}$. Their zeta potential is $+48 \mathrm{mV}$ in deionized water, which stands for a high stability. Concentration of 
particles in water was measured from iron concentration with UV-visible spectroscopy Cary 50 Varian at $500 \mathrm{~nm}$. A linear correlation was found between particle concentration and absorbance in the 0 to $0.06 \%$ volume concentration range with an uncertainty of $3 \mu \mathrm{mol} / \mathrm{L}$ of iron.

\section{Transport experiments}

Each sand column was installed vertically in the MRI core. The column bottom was linked to a peristaltic pump, and the column top to a fraction collector (out of the Faraday cage) via Tygon tubes of $1.52 \mathrm{~mm}$ inner diameter and $4 \mathrm{~m}$ long (see Figure 1). We checked that particles did not adsorb on tube walls or in filters.

Pure water was first flowed through the system until the volume of water in the column was twice renewed. Experiments were carried out at three different Darcy fluxes (flow rate per sample unit section area): $Q=12,42$ and $60 \mu \mathrm{m} / \mathrm{s}$. Then, a given volume (between 18 and $43 \mathrm{ml}$ (see Table 1)) of particle suspension of $3.5 \times 10^{-4} \mathrm{~mol}$ of $\mathrm{Fe} / \mathrm{L}$ (corresponding to a volume fraction of particles of 0.053 $\%)$ was injected, and pure water was injected again. This operation, i.e. pure water flow - particle injection - water flow, was repeated several times (up to five times) for each column. In this context the injections are numbered in the order of the tests. The characteristics of the three experiments are described in Table 1. The outputs were collected continuously during the whole experiment with a fraction collector. Straining (geometrical jamming of particles due to small pore size) was likely negligible in our experiments because the ratio of colloid diameter to collector mean diameter is equal to 0.00016 , which is much smaller than the ratio of 0.0017 reported by Bradford et al. (2002) for significant colloid straining. Some authors also found in specific cases that a significant straining could occur for a ratio as low as 0.0002 (Tosco and Sethi 2010; Raychoudhury et al. 2014). However we have a straightforward evidence that straining was negligible in our case: experiments of injection of the same type of particles but here negatively charged (so that adsorption was not allowed) through the same type of porous medium, were recently carried out and a negligible amount of blocking was observed (Lehoux et al. 2016, PRE). 


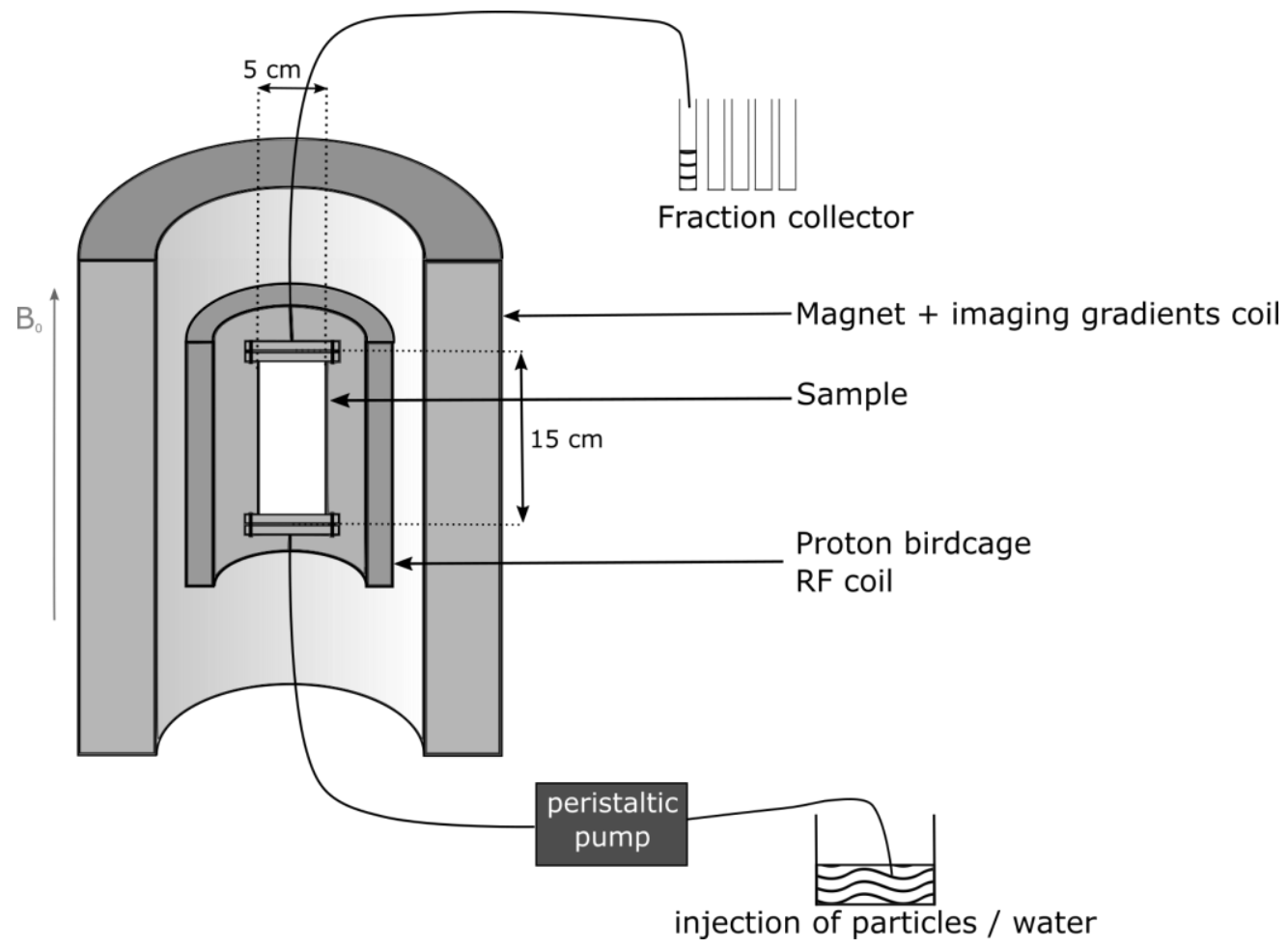

Figure 1 : Scheme of the setup.

\begin{tabular}{|c|c|c|c|c|c|}
\hline & $\begin{array}{c}\text { Injection } \\
\text { number }\end{array}$ & units & A & B & C \\
\hline Concentration & all & $\mathrm{mol} / \mathrm{L}$ & $3.5 \times 10^{-4}$ & $3.5 \times 10^{-4}$ & $3.5 \times 10^{-4}$ \\
\hline Darcy Flux & all & $\mu \mathrm{m} / \mathrm{s}$ & 11.9 & 41.9 & 60.3 \\
\hline \multirow{4}{*}{ Injected volume } & $\mathbf{1}$ & $\mathrm{ml}$ & 28.9 & 24.8 & 30.3 \\
\cline { 2 - 6 } & $\mathbf{2}$ & $\mathrm{ml}$ & 28.0 & 29.8 & 29.7 \\
\cline { 2 - 6 } & $\mathbf{3}$ & $\mathrm{ml}$ & 42.6 & 24.5 & 30.0 \\
\cline { 2 - 6 } & $\mathbf{4}$ & $\mathrm{ml}$ & & 29.7 & 29.9 \\
\cline { 2 - 6 } & $\mathbf{5}$ & $\mathrm{ml}$ & & 29.6 & 17.8 \\
\hline
\end{tabular}

\section{MRI measurements}

MRI experiments were carried out with a vertical imaging spectrometer DBX $24 / 80$ by Bruker operating at $0.5 \mathrm{~T}(20 \mathrm{MHz}$ proton) and equipped with a birdcage radio frequency (RF) coil delimiting a measurement zone of $20 \mathrm{~cm}$ inner diameter and $20 \mathrm{~cm}$ height. The apparatus is exclusively sensitive to hydrogen atoms $\left({ }^{1} \mathrm{H}\right)$ which are in the present case almost exclusively those of water because of the very short $T_{2}$ relaxation time of its components. 
In a standard MRI measurement the spins of the protons of the hydrogen atoms are excited by a radiofrequency pulse over a short time and then relax to their initial state more or less rapidly depending on their Nuclear Magnetic Resonance (NMR) relaxation times. In our tests a NMR signal $S$ detected at a detection time $\tau$ reads (Callaghan (1991)):

$S(\tau)=S_{0} \exp \left(-\frac{\tau}{T_{2}}\right)$

where $T_{2}$ is the transverse relaxation time and $S_{0}$ (in arbitrary units) the NMR signal amplitude before relaxation starts, which is proportional to the water amount.

During our experiments, the water content inside the column does not change significantly, which means that the NMR signal amplitude will not change significantly, but suspended or adsorbed particles may be detected through their influence on the relaxation of water molecules. In unconfined conditions (liquid volume without boundaries), suspended particles induce a decrease of the spin-spin relaxation time in water depending on their concentration $C$ proportional to their magnetic property characterized by their relaxivity $R$ (Brownstein and Tarr, 1977) such that

$\frac{1}{T_{2}(C)}=\frac{1}{T_{2, b u l k}}+R \times C$

in which $T_{2, b u l k}$ is the transverse relaxation time in -particle free- bulk water, $C$ the particle concentration and $R$ their relaxivity, which is a factor depending on the surface characteristics. At a given temperature and working magnetic field, $R$ is a constant, and may be determined from independent measurements on suspensions of known concentrations. In that aim we measured $T_{2}$ for a suspension volume of $1 \mathrm{~cm}^{3}$ for various concentrations of suspended particles with a table spectrometer Minispec MQ20 ND-Series by Bruker operating at the same magnetic field and frequency than the vertical imaging spectrometer. This equipment allows precise measurements for small samples. Figure 2 shows the corresponding results, from which we deduce $R \approx 101.5 \mathrm{mmol}^{-1} \cdot 1 . \mathrm{s}^{-1}$.

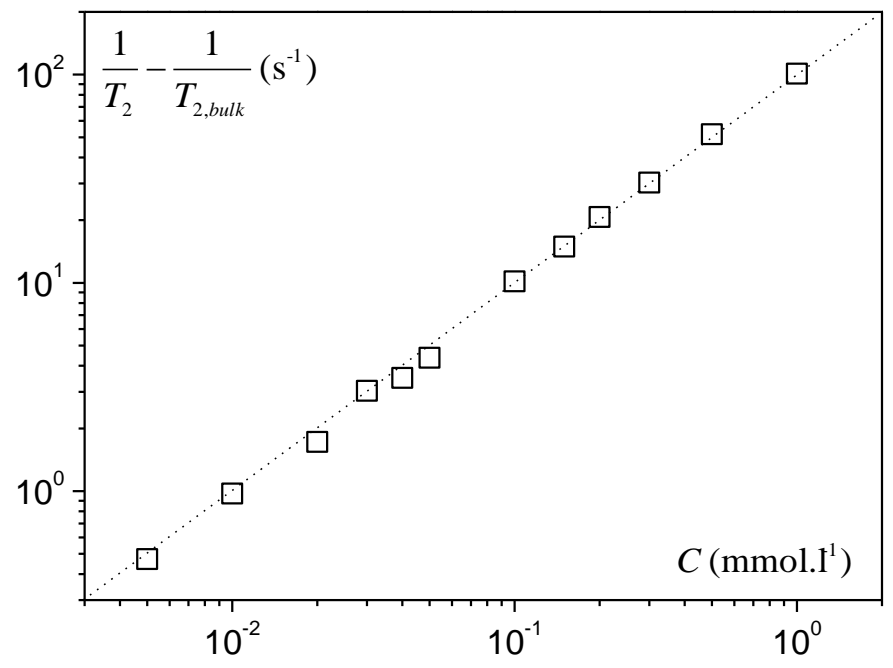



liquid (pure water). The dotted line is a line of slope 1 (in logarithmic scale) fitted to data, from which we deduce the value of $R$ through eq. (2).

When pure water is embedded in a porous media, physical interactions with pore surface provide another source of enhanced relaxation, which may be described with the help of a factor, namely the surface relaxivity $\rho$ (in $\mathrm{m}_{\mathrm{s}} \mathrm{s}^{-1}$ ), which depends on the characteristics of the system. In a water saturated porous medium, the influence of $\rho$ on water relaxation is governed by the ratio $\rho d / D_{w}$ (Brownstein and Tarr, 1977), where $d$ is a typical pore diameter, and $D_{w}$ is the self-diffusion coefficient of water $\left(2.10^{-9} \mathrm{~m}^{2} \mathrm{~s}^{-1}\right.$ at $\left.20^{\circ} \mathrm{C}\right)$. When this ratio is much smaller than unity, the relaxation is said to be surface-limited, and results in an apparent decrease of $T_{2}$ in water, according to:

$\frac{1}{T_{2}(\rho)}=\frac{1}{T_{2, b u l k}}+\rho \frac{A}{V}$

$A / V$ is the surface area to pore volume ratio of the pores where water is located. In other cases (i.e. when $\rho d / D_{w}$ is not much smaller than unity) the relaxation process becomes more complex and the NMR signal at $\tau$ reads:

$S(\tau)=S_{0} f(\tau, \rho)$

in which $f$ is a decreasing function of $\rho$; for a given surface relaxivity, $f$ is a sum of various positive decreasing exponential functions of $\tau$. For $\rho d / D_{w}>>1$, the dependence on $\rho$ is finally lost, and the relaxation is said to be diffusion-limited.

Adsorbed particles can be detected because they modify the surface relaxivity of the grains (Bryar et al. 2000; Keating and Knight 2007) as a function of their concentration $s$ (in moles per gram of dry sand). The water at the contact of adsorbed particles relaxes differently than in the presence of suspended particles, because relaxation on a surface covered with paramagnetic centers is governed by different physics (Korb et al. 2007). $\rho(s)$ is expected to be an increasing function of $s$. A linear relation was observed in some systems for low adsorbed concentration (Bryar et al. 2000), but to our knowledge there is still no theory nor convincing experiment extending this linearity up to saturation level. This lack of knowledge is skipped in our study. Indeed, due to strong adsorption expected from our particles, only two cases will be considered in further interpretations: either the sand grain is free of adsorbed particles, or it is fully covered. In the absence of suspended particles, the NMR signal at $\tau$ can be rewritten:

$S(\tau)=S_{0}\left[\exp -\left(\frac{1}{T_{2, \text { bulk }}}+\rho_{0} \frac{A}{V}+R_{\text {ads }} s\right) \tau\right]$

where $\rho_{0}$ is the surface relaxivity with no adsorbed particles (relaxation was checked to occur in surface limited regime in this case), and $R_{a d s}\left(\right.$ in $\mathrm{s}^{-1}$ ) is a pseudo-relaxivity constant only defined so 
that $R_{a d s} \times s$ is the contribution of adsorption to relaxation when the sand surface is saturated with particles, no matter the relaxation regime.

In the presence of both adsorbed and suspended particle, the relaxation terms associated with suspended species and surface effect are added (Keita et al. 2013), and the NMR signal at $\tau$ finally expresses as:

$S(\tau)=S_{0}\left[\exp -\left(\frac{1}{T_{2, \text { bulk }}}+\rho_{0} \frac{A}{V}+R C+R_{a d s} s\right) \tau\right]$

In order to detect particles, we used MRI protocols sensitive to relaxation times. For quantitative measurements, $T_{2}$-weighted $1 \mathrm{D}$ profiles imaging along longitudinal $z$ direction of the sample, i.e. parallel to water flow, were measured with a spatial resolution of $1.56 \mathrm{~mm}$. The sample is observed projected on $z$ axis, and the signal recorded in a 1D pixel is that of the whole cross sectional layer projected on the pixel.

The MRI sequence used was a double spin-echo (two first echoes of CPMG sequence (Carr and Purcell 1954; Meiboom and Gill 1958)) with echo time $T_{\mathrm{E}}=3.32 \mathrm{~ms}$, repetition time $T R=7 \mathrm{~s}$, and with a read-out imaging gradient superimposed over each echo. Only the second echo (detection time $\tau=2 T_{\mathrm{E}}=6.64 \mathrm{~ms}$ ) was taken into account, as it was found to bring optimized sensitivity to contrast agent. The signal was accumulated over 8 scans, and double spin-echo profiles of the entire sample were recorded every $65 \mathrm{~s}$. Considering the range of average velocities of fluid through the pores (mean velocity divided by porosity), the mean distance covered by a fluid element during one acquisition ranges from 2 to $10 \mathrm{~mm}$. This implies that our spatial resolution is of the order of $2 \mathrm{~mm}$ for the lowest velocities and of the order of $10 \mathrm{~mm}$ for the highest ones.

Since the actual distribution in the cross-section can be inhomogeneous, collected NMR signal in a pixel reads:

$S(z)=S_{0}(z)\left\langle\exp -2 T E\left(\frac{1}{T_{2, \text { bulk }}}+\rho_{0} \frac{A}{V}+R C(x, y, z)+R_{a d s} s(x, y, z)\right)\right\rangle$

$S_{0}(z)$ represents the water signal intensity in a pixel before relaxation starts. Since the volume fraction of particles remains small whatever the evolution of adsorbed or suspended particle amounts, $S_{0}(z)$ is regarded as a constant over the whole experiment. The brackets stand for an averaging in transverse directions at a fixed $z$ coordinate. Let $S_{r e f}(z)$ be the MRI signal measured without particles. If $T_{E}$ remains small regarding actual relaxation contribution of particles, then information on particles only can be approximated by

$$
\frac{1}{2 T_{E}} \ln \left(\frac{S_{r e f}(z)}{S(z)}\right) \approx R_{a d s} \times<s>(z)+R \times<C>(z)
$$

The right hand term in this equation is a linear combination of suspended and adsorbed projected concentration profiles, and can be further interpreted depending on the experimental context. 
The constant $R_{a d s}$ is calibrated with eq. (8) from MRI data after the last injection in each column assuming that sand is covered by particles up to its maximum amount (inferred from the difference between the amount of particles injected and detected at the output), and no suspended particles are left in the sample. Let us emphasize again that this constant is not intended to estimate relaxation effects at intermediate $s$ values. We found similar values for each column with a standard deviation of about $16 \%$ (average value of $R_{\text {ads }}=4.5 \times 10^{8} \mathrm{~g} \cdot \mathrm{s}^{-1} \cdot \mathrm{mol}{ }^{-1}$ ).

As mentioned by Ochiai et al. (2006), MRI can detect particles only if the injected concentration is high enough. For example in our study, and without adsorption, $10^{16}$ of these particles $\left(=1.5 \times 10^{-4} \mathrm{~mol}\right.$ $\mathrm{Fe} / \mathrm{L})$ are required to make $S(z)$ and $S_{\text {ref }}(z)$ differ by $10 \%$. We used somewhat higher concentrations (see table 1) to get a clearer effect, while remaining in the range of validity of eq. (8). The typical uncertainty on average local concentrations due to the approximation in (8) was kept at the same level as experimental noise owing to finite signal to noise ratio on raw NMR signals, and were found to be $\pm 1 \times 10^{-5} \mathrm{~mol} \mathrm{Fe} / \mathrm{l}$ for suspended concentration and $2 \times 10^{-9} \mathrm{~mol} / \mathrm{g}$ dry sand for adsorbed concentration. Additional bias could also arise from thermal fluctuations of hardware characteristics -especially RF coil sensitivity- over long measuring time, and lead to a global vertical shift of concentration profile. This effect was found to be very small in the present study, but we believe it is at the origin of slight baseline imperfections sometimes visible in further graphics. At last, sample areas close to upper and lower limits of the measuring zone were disregarded, due to locally depreciated signal to noise ratio. Profiles presented in the following are then limited to a 14 $\mathrm{cm}$ wide central zone of the sample, containing 90 pixels.

Since profile measurement may hide an inhomogeneous distribution of particles in transverse directions, 2D MRI images of a $5 \mathrm{~mm}$ thick vertical slice passing through the middle of the column with space resolution 1.46 (transverse) $\times 1.56$ (longitudinal) $\mathrm{mm}$, were taken after each injection. A Spin Echo Imaging sequence was used $\left(T_{E}=6.54 \mathrm{~ms}, T R=2 \mathrm{~s}\right)$. Short $T R$ value was chosen so as to keep measurement time under 5 minutes ( 4 minutes and 25 seconds). It however induces both $T_{1^{-}}$and $T_{2^{-}}$ weighting, which makes signal dependence on particle concentration more complex, thus preventing in our case particle quantification through a simple comparison with a reference. $2 \mathrm{D}$ images should then be seen of qualitative interest only, and cannot be directly compared with 1D profiles.

In short, this MRI study is based on time-resolved measurements of total concentration profiles (showing both suspended and adsorbed particles) during the flow, and profiles showing only adsorbed particles at the end of each pulse injection. Images of adsorbed particles recorded at the end of each injection are used for qualitative interpretation.

\section{Results and discussion}

Once injected, the suspension of particles flows through the tube towards the column. In the column, particles may either remain suspended or get adsorbed onto the grains. However we observe that in any case, after each of the first three injections, no particles are detected at the output during a flow time corresponding to the exit of three times the total pore volume of the column. This means that for each of these tests all the particles have been adsorbed onto the grains. 

information about larger time-scales.

After the next injections, some particles are detected at the output (except for column A where only 3 injections were performed and all particles were adsorbed to the porous media). In column B, particles are only detected after the $5^{\text {th }}$ injection, up to $16 \%$ of injected particles. In column $\mathrm{C}$, as the injections were slightly larger (see injected volumes in table 1), $28 \%$ of injected particles were detected at the output after the $4^{\text {th }}$ injection, and $63 \%$ after the $5^{\text {th }}$. This means that adsorption occurs until approximately $100 \mathrm{ml}$ of solution of particles at $3.5 .10-4 \mathrm{~mol} / \mathrm{L}$ injected.

If we assume that for the tests $B$ and $C$ adsorption has reached a maximum in every point in the column, we can compute the related amount of particles adsorbed on each sand grain, by subtracting the amount of particles detected at the outputs from the particles injected in the system, and dividing this by the total mass of sand in the column. We find for column $\mathrm{C}$ an average value of $7.8 \times 10^{-8} \mathrm{~mol}$ per gram of dry sand and for column $B$ a value of $8.7 \times 10^{-8} \mathrm{~mol}$ per gram of dry sand. In order to study the process more precisely we first focus on the successive final adsorption profiles obtained after each series of injections. Then we study the transient processes, i.e. particle motion and stoppage during a given injection.

\subsection{Adsorption}

The profiles of adsorbed particles after successive injections for the tests at different velocities are shown in figure 3. Here we focus on profiles obtained after the first three injections. They look qualitatively similar and their basic trends are as follows: each profile starts with a plateau from the entrance of the column and finishes by a front along which the particle concentration rapidly (over 1 to $2 \mathrm{~cm}$ ) decreases to zero; from one injection to the next one, the length of this plateau increases while the front advances, keeping almost the same shape. The level of the plateau does not evolve from one injection to the other in test $B$, and shows very limited evolution in test $C$. Actually these successive profiles suggest that particles advance and rapidly occupy -almost-all possible sites of adsorption on the grains; after the injection there is a region (plateau) in which no more particles can be adsorbed, and the next particles arriving behind have to advance farther to find new free sites.

Let us now look at these profiles in more details. First of all we can remark that the plateau is not perfectly flat: apart from the fluctuations over short lengths which are due to noise on measurements there are also slight variations over larger lengths. The latter are likely due to heterogeneities of the column packing, leading to local variations of the specific surface area and thus of the number of adsorption sites available. This is confirmed in tests $A$ and $B$ by the fact that the profiles after successive injections very well superimpose in the regions where the maximum amount of adsorbed particles has been reached. For the highest velocity (C) however, the final plateau level is not reached immediately after the first injection, the next injections induce a minor but clearly visible progressive increase of the plateau level while the front still significantly advances more or less similarly as for the other tests. This suggests that at low velocities, particles have sufficient time to explore the pores and finally fill all available sites existing around them whereas at sufficiently high velocities they miss some sites and go on advancing. This point will also be discussed farther in this paper. 
Let us now focus on the values of maximum of absorption. Due to the calibration procedure of $R_{\text {ads }}$, the plateau values in Figure 3 are simply those deduced from the macroscopic analysis (see above): the average value of coverage for $A$ at the plateau is $5.8 \times 10^{-8} \mathrm{~mol} / \mathrm{g}$ dry sand, for $B$ of $8.9 \times 10^{-8} \mathrm{~mol} / \mathrm{g}$ and for $C$ of $7.1 \times 10^{-8} \mathrm{~mol} / \mathrm{g}$, leading to an average value of $7.3 \times 10^{-8} \pm 8 \% \mathrm{~mol} / \mathrm{g}$, the uncertainty likely being due to packing heterogeneities. As far as the interpretation of raw NMR data is concerned, let us emphasize that $S / S_{\text {ref }}$ ratio (see eq. 8) was about 0.8 in the saturated zone. Had NMR relaxation occur in surface limited regime, and taking $\frac{6}{d_{\text {grain }}} \frac{1-\varepsilon}{\varepsilon}$ as the surface to volume ratio of the pore space (assuming spherical sand grains, with $\mathrm{d}_{\text {grain }}$ the average diameter of sand grains) this corresponds to a surface relaxivity of $\rho=7.10^{-4} \mathrm{~ms}^{-1}$. Thus a Brownstein and Tarr criterion roughly equals to $\rho d_{\text {grain }} / D_{w} \approx 60>>1$, which finally contradicts the first hypothesis stating that the relaxation was surface-limited. This brings an a-posteriori support for considering all various relaxation regimes in data analysis, and not only the surface limited case.

From the maximum average value for adsorption $\left(7.3 \times 10^{-8} \mathrm{~mol} / \mathrm{g}\right.$ dry sand $=4.9 \times 10^{12}$ particles per gram of dry sand), and assuming that the sand grains are spherical, the average surface coverage is then $59 \%$ with a variation of $\pm 3 \%$ between all tests. Note that the actual uneven shape of sand grains as shown in Jacobs et al. (2007), would probably lead to an increased grain surface and thus a slightly lower coverage value.

As no desorption is observed on MRI profiles nor measured at the end of the column during the flow, it is natural to consider that particles do not leave the grain surface when they have been adsorbed somewhere over our time-scale of observations. The question of how particles occupy and potentially reorganize themselves on the surface after adsorption can be discussed considering the following reference values. The maximum packing of solid discs (which is a situation equivalent to spheres over a planar surface) is $91 \%$ for a close-packing disposition (Israelachvili 2011). However the maximum random close-packing implies excluded surfaces, and is reported to be about $82 \%$. In this distribution the discs are disordered and have more than one point of contact with each other, which suggests that the effective maximum packing fraction obtained by successive adsorption of particles unable to move to pack more efficiently, is significantly lower than this value. Adamczyk (2000) precisely studied the situation of hard spheres with a Random Sequential Adsorption (RSA) model where spheres keep their position after adsorption, and calculated a value of maximal coverage of 54.7\% (this model was also used for colloids in porous media by Johnson and Elimelech (1995) and Ko et al. (2000)).

This result is rather close to our experimental value $(59 \% \pm 3 \%)$, which suggests that the adsorption of our particles could essentially follow a RSA mechanism, with no possible motion of particles after adsorption. There might also be an effect of repulsion of the adsorbed particles on the approaching particles due to their positive electric charge, which would tend to decrease the probability of reaching some free surface region of the grains. Granted these adsorption conditions, we regard thus the found surface coverage as representative of some surface saturation with one monolayer of adsorbed particles. Note that in that case they form approximately a layer of extremely small thickness as compared to the pore size (the ratio of particle to grain size is of the order of 0.0001 ), which means that the impact on the available volume is negligible. Then the basic change of 


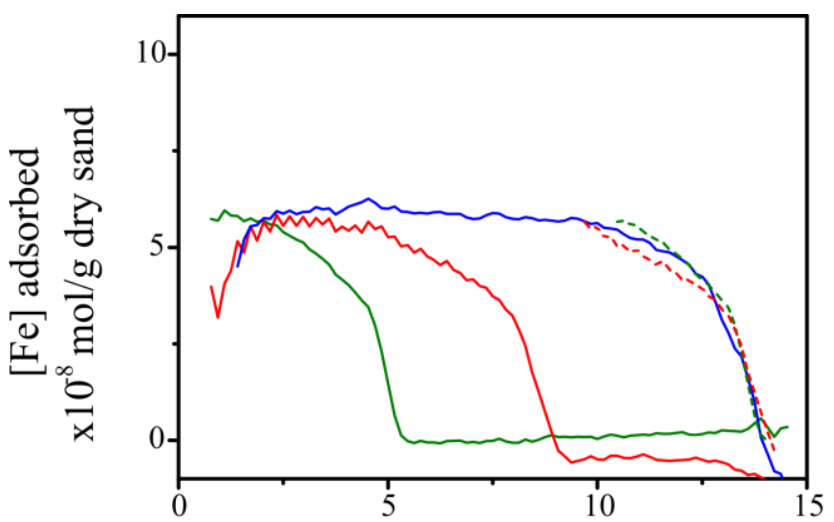

(a)

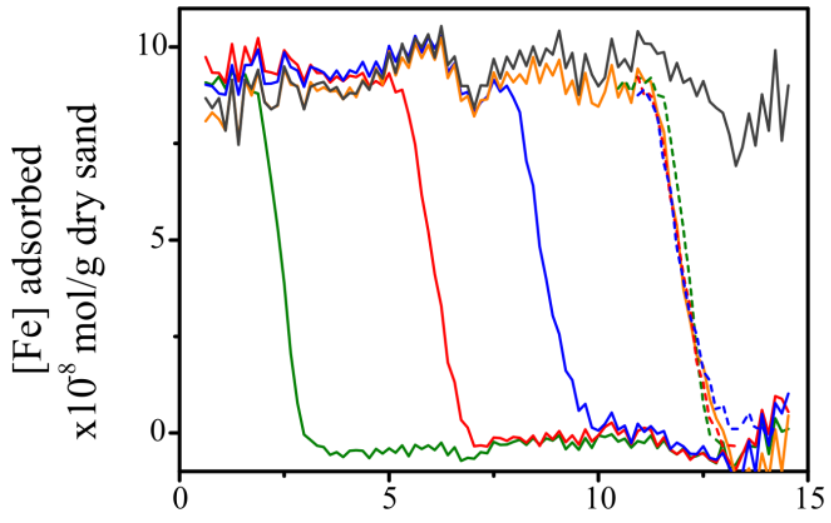

(b)

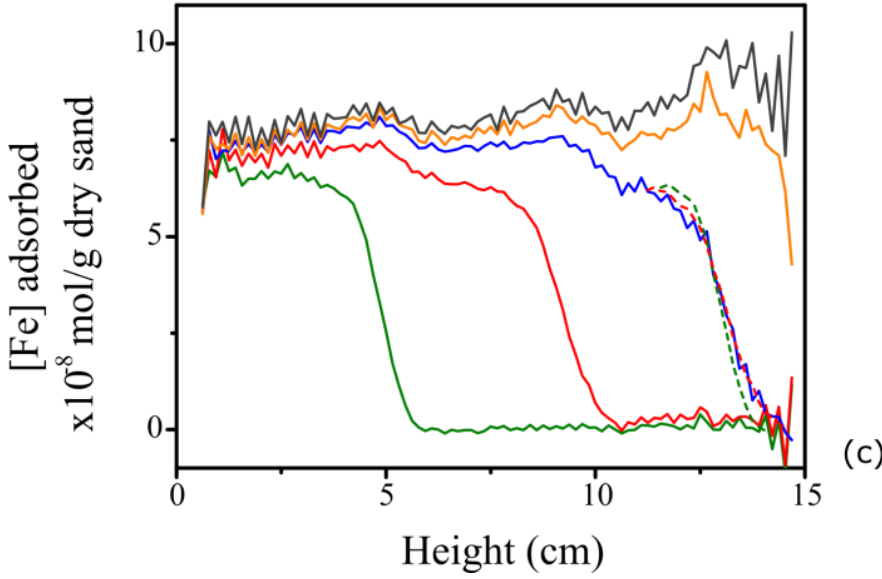

Figure 3: Adsorbed concentration profiles (from MRI) after the successive injections (1: green, 2: red, 3: blue, 4: orange, 5: black) for the tests at different Darcy Flux: (a) $A=11.9$ $\mu \mathrm{m} / \mathrm{s}$, (b) $B=41.9 \mu \mathrm{m} / \mathrm{s}$, (c) $C=60.3 \mu \mathrm{m} / \mathrm{s}$. Profiles are estimated from eq. (8) assuming no suspended particles are present. Slightly negative values may occur as a result of baseline fluctuations and noisy raw NMR data. Dashed lines correspond to a shift of front from profiles obtained at the other injections onto the $3^{\text {rd }}$ or $4^{\text {th }}$ profile on purpose of shape comparison.

It seems then that the major mechanism underlying our observations is as follows: after the different injections, particles get adsorbed onto the grains until (close to) total coverage. When an additional amount of pure water equivalent to the total pore volume is flowed, adsorbed profiles remain 
unchanged, i.e. particles are fixed in the column. This is consistent with the assumed strong adsorption behavior of particles used in this experiment -and confirms a posteriori this behaviour-, and also with observation (see previous section) that no particles are observed in the output after the first three injections. This also a priori implies that when a particle is adsorbed somewhere it will not be removed later by the flow.

We now look at the shape of fronts. Fronts of the different injections of the same test are similar. Indeed they very well superimposed when the profiles are shifted by an appropriate distance (see Figure 3). This means that despite the complexity of the flow and the adsorption processes, which take place during the successive injections, the spatial progression of particles remains the same at each step. This could suggest that this shape results from the dynamics of the process: even if we have seen that particles rapidly adsorb as soon as they reach regions with available sites, some of them could advance farther before encountering such sites, which would yield a partially saturated front with an inclined concentration profile - Note that in such situation, since the theory of NMR relaxation in partially saturated zones is not established, front regions in figure 3 should be regarded as only qualitative - . However had such effect be characterized by a specific time $T$ for a suspended particle to find an adsorbing site in the particle-free area, then, from a dimensional analysis, the slope should scale as $T / Q$, i.e. the slopes in test $A$ should be higher than in test $C$ by a factor of 6 , which is not clearly the case here. This suggests that the front shape has likely another origin.

In fact the front shape might find its origin in the heterogeneities of the columns since profiles are obtained by averaging the data over cross-sectional layers, which hides information on particle distribution in transverse directions. To clarify this aspect we can look at MRI images obtained after each injection (see Figure 4): we indeed observe a front with uneven shape. As a consequence, the front of adsorbed particle concentration is also uneven. For a series of injections of a same test, these irregular fronts are rather similar from the first injection to the last one. As expected from the longitudinal profiles they are however different for different tests. The shape of this front of particles is then likely to be mainly ruled by the configuration of the flow at the injection point in the column (Lehoux et al.2016), as this configuration is kept constant until the end of the test. The simple translation of the front shape along the sample axis means that, as soon as it has been formed around the entrance of the sample, the fluid flows essentially parallel to the sample axis and at a uniform average velocity so that the front shape is kept constant.

Finally we can consider that the shape of the longitudinal profile at the front results mainly from the uneven 3D shape of a sharp flow front in the column. This is confirmed by a rough estimation of the front lengths from the pictures of Figures 3 and 4: for test $A$ the front has a length of $3 \mathrm{~cm}$ in the profiles and an irregular shape in the MRI images of about $2.8 \mathrm{~cm}$. For test $B$ it varies from 1.1 to 2 $\mathrm{cm}$ in the profiles while it varies from 1.3 to $2.5 \mathrm{~cm}$ in the images; and finally for test $\mathrm{C}$ it ranges between 1.6 and $2.5 \mathrm{~cm}$ in the profiles, and between 1.4 and $2.1 \mathrm{~cm}$ in the images. We can note that the lengths in the profiles are larger than the lengths on the images (except for column B), an effect which might be attributed to the fact that the images only show the distribution of particles in a specific longitudinal cross-section of $5 \mathrm{~mm}$ while some slightly larger irregularities can be expected in other transverse directions, and show in the profile measurements.

Liquids are heterogeneously disposed in the column when they enter, and this heterogeneity is then kept constant all along the column. This shows that the flow in the column is homogeneous. 
Particles are strongly adsorbed. Adsorption occurs up to an average of $7.3 .10^{-8} \mathrm{~mol}$ of Fe per gram of sand corresponding to $59 \%$ of coverage of the grains. This means that particles are randomly disposed on the grains surface.

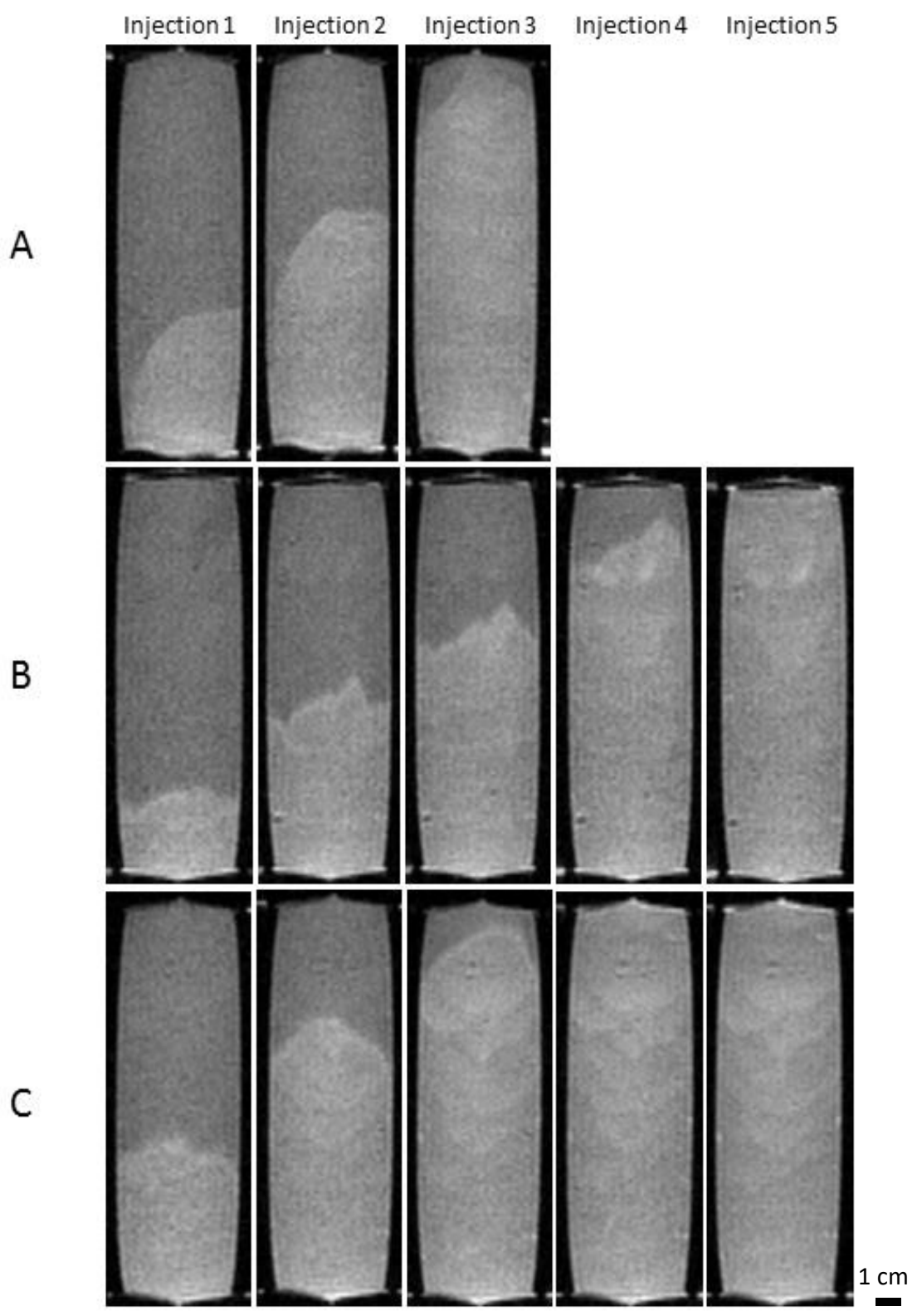

Figure 4: MRI images of the repartition of adsorbed particles (white) after each injection in the 3 columns (length: $15 \mathrm{~cm}$ ).

\subsection{Transport}

436 Let us now focus on the flow dynamics, i.e. the process by which suspended particles get adsorbed 437 during a given injection. Typical 1D profiles measured during this process are shown in Figure 5 . Such 438 measurements correspond to the combinations of suspended and adsorbed particle effects on the 439 signal, as described from eq. (8). 
Since the relative weights (on the NMR signal) of adsorbed and suspended particles are different, the interpretation of profiles is not as straightforward as when there are only adsorbed particles in the sample. We can nevertheless identify several trends of the process by deduction, in particular by following the successive evolutions of the total profile. Here we carry out such a complete analysis on the profiles for test $B$ (see Figure 5) but similar results leading to similar analysis were obtained for the two other tests.

For the first injection (see Figure 5a) we see that as expected there is an overall particle transport towards the end of the column: the profiles globally spread along the flow direction. Since we $a$ priori ignore when particles are getting adsorbed we cannot know the fraction of particles still in suspension at a given position. The final profile corresponds to that described in previous section, namely a plateau and a short front.

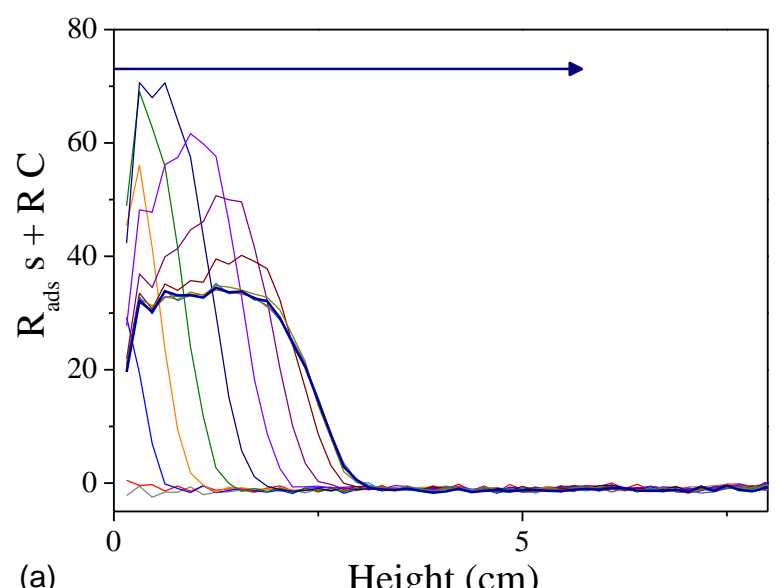

(a)

$$
\text { Height }(\mathrm{cm})
$$

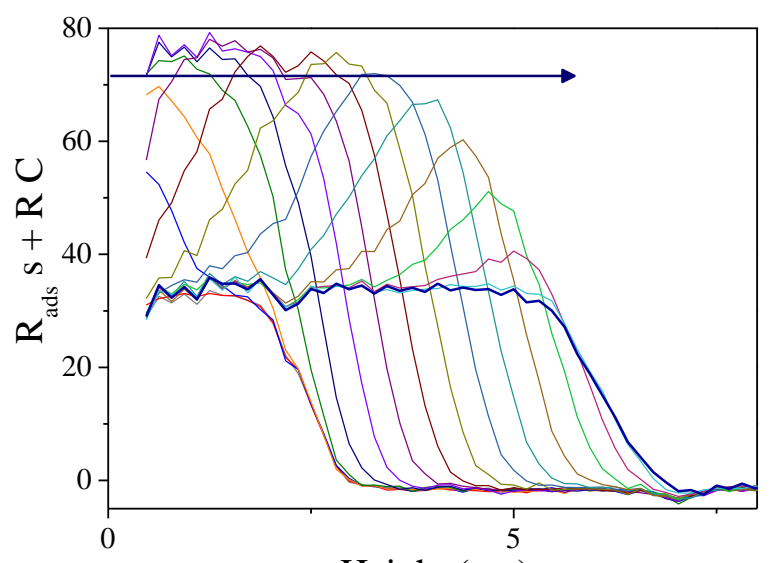

(b)

Height (cm)

The situation is different for the second injection (see Figure 5b). Since there is a region where the

Figure 5: Combined adsorbed and suspended concentration profiles (as deduced from eq. 8) during particle transport through the column B compared to the profile without particles: (a) injection 1, (b) injection 2. maximum concentration of adsorbed particles has been reached, we can conclude that in this region 
the profile is the -weighted- sum of the saturated adsorption profile (which is known) and that of suspended particles. The suspended particles are now transported farther in the column where adsorption sites are available. This leads to an increase of the plateau length as described in previous Section. A similar process takes place during the next injection, eventually leading to a further increase of the plateau length. Now let us look at the measured total concentration level at one given position along the column axis where there are initially no adsorbed particles. In the pre-existing region of adsorbed particles - area already covered with adsorbed particles - the signal first increases then decreases with a shape resulting from the injected pulse, and this shape is kept approximately constant along this pre-adsorbed region (see the two curves at the shortest distance along the column in Figure 6). In the neighboring region where particles had not already been adsorbed after the first injection, the measured total profile increases at a lower level and then decreases to the plateau value; in the region situated at a larger distance it remains equal to zero: the particles do not reach it.

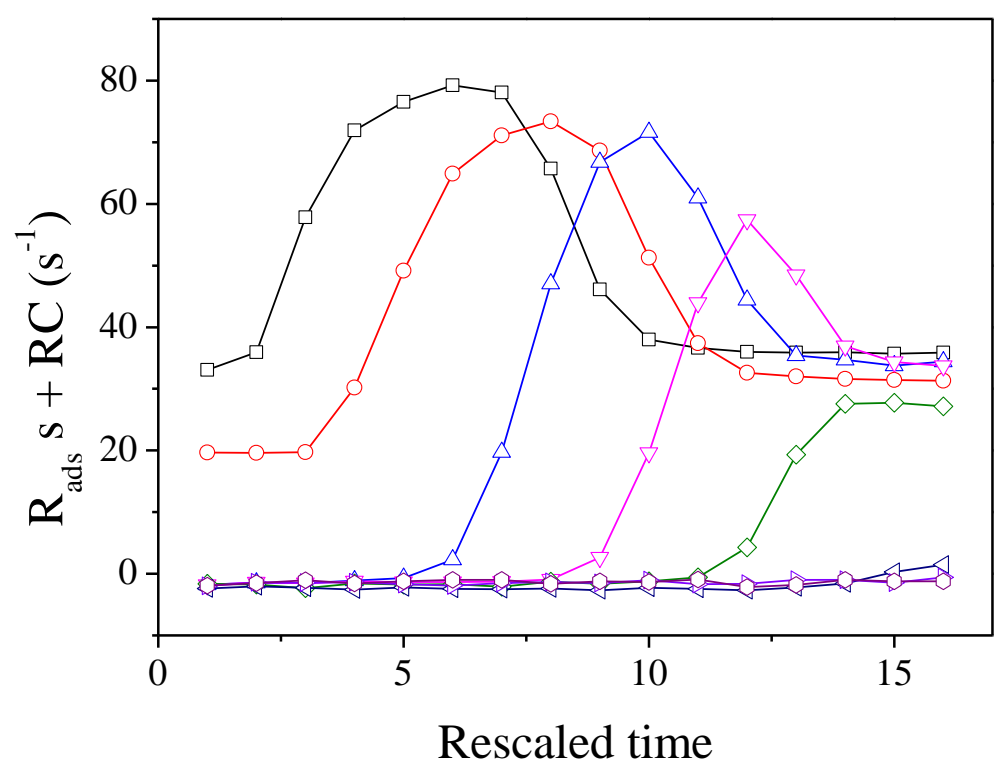

Figure 6: Time evolution of the combined adsorbed and suspended concentration profiles (as deduced from eq. 8) at different positions along the column for the second injection in column B: (from top to bottom) 1.2, 2.3, 3.4, 4.5, 5.6, 6.7, 7.8, $8.9 \mathrm{~cm}$.

Since we are dealing with nanometric particles, sedimentation effects are negligible in our tests. Since the volume fraction of particles in the liquid is very small $(0.053 \%)$ the suspension behaves as a Newtonian fluid of viscosity close to that of water, and the flow properties are governed by the liquid (i.e. the suspended particles do not play any role on the flow characteristics). The Reynolds number inside the porous medium is $\operatorname{Re}=\rho_{w} v d / \mu$, where $\rho_{w}$ is water density, $v=Q / \varepsilon$ is the mean velocity through the pores, $\mu$ the fluid viscosity and $d$ the characteristic diameter of the pore, which can be calculated from $d=d_{\text {grain }} \varepsilon /(3(1-\varepsilon))=48 \mu m$ (Scheven 2010). Finally in our range of flow rates $R e$ is in the range $[1.5-7.5] \times 10^{-3}$ which means that the flow is laminar. This implies that flow characteristics, in terms of streamlines in particular, are strictly similar for our three tests. 
Nevertheless the colloids can diffuse in the liquid under the action of thermal agitation, so that different flow rates may leave different times for particles to explore the pores.

Following Stokes-Einstein formula, the diffusion coefficient of the particles is $D_{m}=\frac{k_{B} T}{6 \pi \mu r}=1.26 \times 10^{-11} \mathrm{~m}^{2} / \mathrm{s}$ with $k_{B}$ the Boltzmann constant, $T$ the temperature, and $r$ the particle radius. In a spherical pore of typical size $d=2 R$ and initially containing an homogeneous concentration of particles, assuming instantaneous adsorption of particles reaching the surface, the time required for $95 \%$ particles adsorption can be calculated from diffusion theory with adsorbing boundary conditions (Crank 1975) and amounts to $T_{D} \approx 0.25 R^{2} / D_{m} \approx 10 \mathrm{~s}$. We regard this value as a typical order of magnitude with our actual pore shape.

The latter time can be converted into a typical distance $l_{\text {ads }}$ that particles need to travel in an unsaturated area before finding an available site where they can get adsorbed: $l_{a d s}=v T_{D}$. One gets $l_{\text {ads }}=300 \mu \mathrm{m}, 1.1 \mathrm{~mm}$ and $1.5 \mathrm{~mm}$ for tests $\mathrm{A}, \mathrm{B}$ and $\mathrm{C}$ respectively. These distances, which may correspond to several grain sizes, remain however very small regarding the scale of our sample and they are of the order of the resolution in our profile measurement. As a consequence, a retardation effect in adsorption kinetic cannot be detected regarding the space resolution of our MRI data. This is consistent with our observation that in our working conditions and observation scale, the plateau of adsorbed particles approximately keeps a fixed level over the successive tests and simply grows in length, the particle just getting almost immediately adsorbed as soon as they encounter pores with some available sites.

Then the particular situation of test $\mathrm{C}$, where adsorption plateau is observed to slightly increase after each new injection, cannot be explained by any retardation due to limited diffusion kinetics.

In this context of favorable adsorption the particles apparently get adsorbed very rapidly when they meet an available adsorption site and they travel farther in the porous medium only when all sites so far have been filled. As already noticed in Lehoux et al. (2016) the particle distribution in the sample essentially depends on their distribution from the entrance. This distribution can be very heterogeneous and will remain similar as all particles will move at the same average speed (beyond some minimum distance) once inside the sample.

\subsection{Distinction of adsorbed and suspended particles}

Now we can carry out a further analysis of the profiles in order to distinguish suspended and adsorbed particles. Since final profiles and images show that adsorbed areas apparently advance by a simple shift of the front (see previous Section), we suggest to consider that this is true for smaller amounts of particle injected at each step. More precisely, under this assumption, any additional small amount of particles entering the column will move towards the front and will finally be adsorbed just beyond the front, and form a similar concentration profile shifted by a small distance.

In this frame we can propose an approach for estimating, from the measured total profile and the adsorbed profile, the distribution of suspended particles in time, according to the following procedure. We neglect changes in plateau level such as those in test $C$, which are regarded, at this 
stage of interpretation, as a secondary effect. This amounts to consider that particles get adsorbed almost instantly when they progress in the column if there are available sites around them, so that during the motion in the column the current distribution of adsorbed particles (red continuous line in Figure 7) simply corresponds to the final one (dark continuous line in Figure 7) shifted (dotted line in Figure 7) in such a way that its farthest point equals that of the current apparent distribution of particles (as observed by MRI). We can eventually deduce the current concentration profile of suspended particles (blue line in Figure 7) by subtracting known adsorbed contribution in eq. (8). The complete procedure is illustrated in Figure 7 for the first injection in column B.

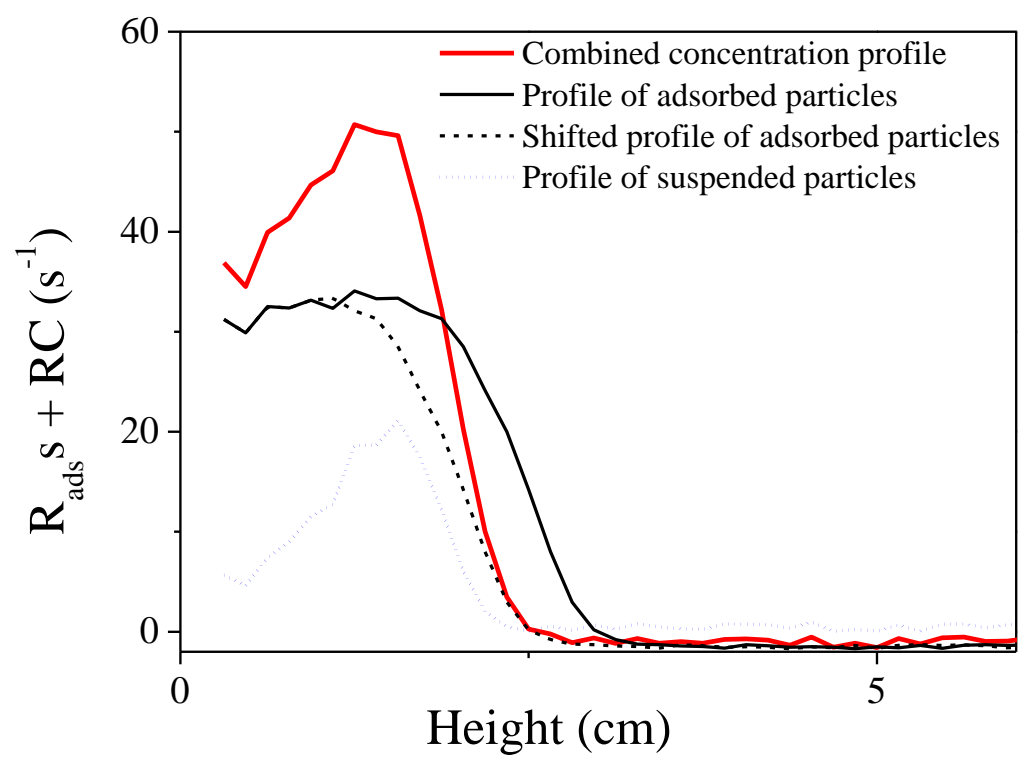

Figure 7: Method of separation of concentration profiles due to adsorbed and suspended particles in column B $6.8 \mathrm{~min}$ after the first injection. The red profile corresponds to the measured total concentration profile.

Using above information concerning suspended and adsorbed particles we can then compare the total amount to the quantity of particles actually injected in the porous media. We measure for each profile $90+/-5 \%$ of injected particles. This result validates our approach; the discrepancy may be explained by the noise on MRI measurements, the noise of visible spectroscopy used to determine the concentration of the initial solution, the potential heterogeneities in the material that can lead to a bias between our theory and the measurements, or some slight deformation of the adsorption front along the sample axis.

Figure 8 shows the evolution of suspended concentration profiles during the $4^{\text {th }}$ injection in column B. The first profiles show that the pulse of particles advances through the column with no visible change of shape because it is transported through the part of the material already colonized by adsorbed particles. From around $9 \mathrm{~cm}$ of the column, the profile amplitude decreases because it has reached the part of the material where sites of adsorption are available. Particles can then get adsorbed on these available sites, and fewer particles are still suspended. We can now shift these profiles by a distance computed as the product of the time elapsed from a reference time and the 
average flow velocity through the pores. By doing so we see how the profile of suspended particles effectively evolves in time (since observations are made in a frame advancing at the speed of the mean flow): the profile first does not evolve (along the initial region of adsorption) then it starts to be progressively eroded along its forefront while the back tail is essentially preserved until all suspended particle disappear (see Figure 9).

We observe a very small dispersion in the suspended profiles when comparing the first profile in the column and the last before adsorption occurs. The pulse of particles is simply transported through the media, following the heterogeneities from the injection point until they can get adsorbed. From a fitting procedure described in (Lehoux et al. 2016), we calculate an average dispersion coefficient $D=6.65 \times 10^{-9} \mathrm{~m}^{2} / \mathrm{s}$. The related ratio $D / D_{m}$, with $D_{m}$ the particle diffusion coefficient, is 0.2 times the Peclet number. It is in excellent agreement with other measured values obtained through direct MRI observation on sand and glass beads packing travelled by non-adsorbing negatively charged particles, and reported in (Lehoux et al. 2016). It is however much lower by almost one decade than coefficients usually reported in literature (Bear 1988; Dullien 1992). An analysis of this apparent discrepancy is out of the scope of the present work, and can be found in (Lehoux et al. 2016).

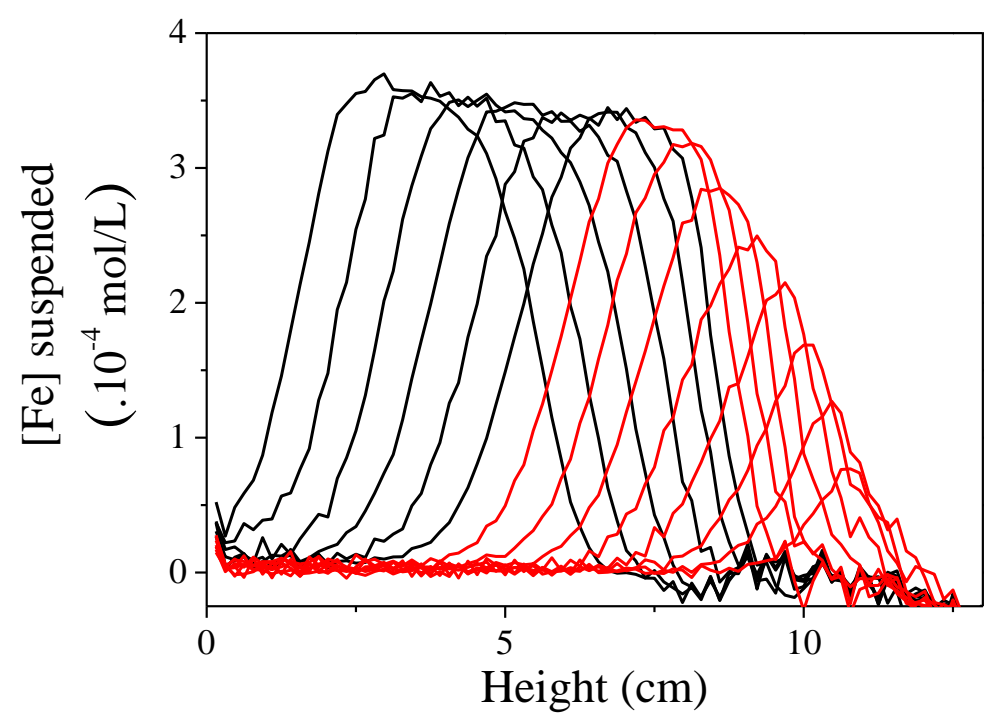

Figure 8: Profiles of iron concentration for suspended particles measured for column $B$, injection 4 (black curves: only transport, red curves: transport and adsorption). 


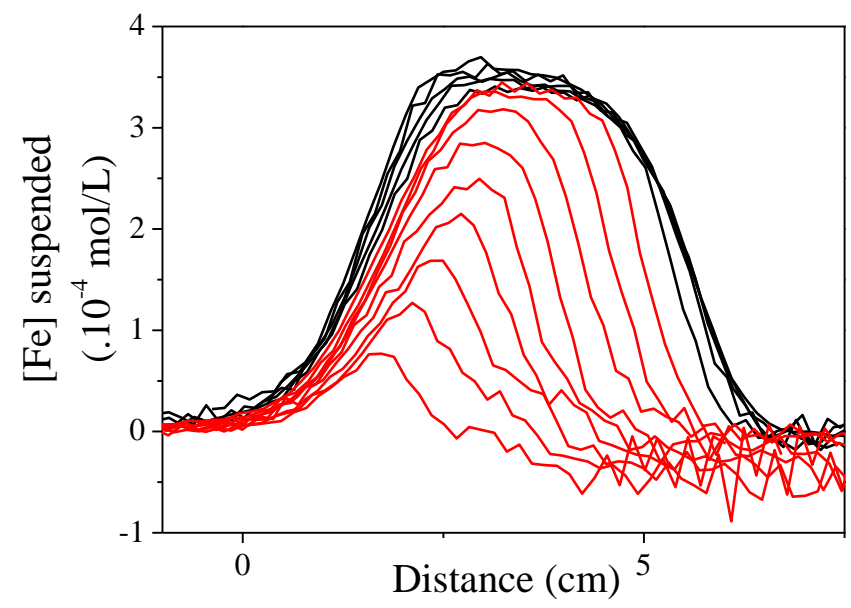

569

570

571

572

573

574

575

576

577

578

579

580

581

582

583

584

585

586

587

588

589

590

591

592

593

594

595

596

Figure 9: Profiles of iron concentration for suspended particles measured for column $B$, injection 4 shifted from a distance corresponding to flow velocity through the pores (black curves: only transport, red curves: transport and adsorption).

In this section we carried out a logical analysis in order to distinguish suspended and adsorbed particles. Then we can follow the transport of suspended particles and show their small dispersion, and the very fast dynamics of adsorption.

\section{Conclusion}

We used MRI to get an internal and dynamic approach of transport and adsorption of positively charged nanoparticles in clean saturated sand. With this technique we could measure a combined signal of both adsorbed and suspended concentrations along the sample length and final profiles and images of adsorbed particles. The latter suggested that particles do not enter homogeneously in the column, but heterogeneities of disposition of adsorbed particles are kept constant through the porous media, showing that particles are transported homogeneously. From this understanding we used a translation of final profiles to calculate concentrations of both suspended and adsorbed particles for each measured total concentration profile measured. We showed that particles get adsorbed as soon as they meet an available adsorption site. When the sand surface is already saturated, incoming particles are transported through the column and can't get adsorbed.

Thus, under our conditions we showed that a wide set of information on the transport dynamics can be obtained from MRI, which can then be analyzed to get a rather precise view of the internal processes. The results obtained here likely concern a specific case (i.e. fast and strong adsorption). For example we can expect that in more complex situations, i.e. when the characteristic time of particle adsorption is larger, only a fraction of particles will be adsorbed at the pulse arrival and significant further adsorption will take place later, while the pulse front has significantly advanced in the sample. Other more complex situations might be considered such as heterogeneous samples (e.g. with complex pore size distributions) implying different characteristic times of adsorption. In 
597 those cases we believe that other, possibly more complex strategies can be developed to analyze the

598 MRI data on the transport dynamics, and possible further NMR techniques for distinguishing

599 adsorbed from suspended particles can be developed, to finally obtain a rich and straightforward

600 information on the internal processes.

601

602 List of Symbols

603 A Surface area of the pores

$604 C$ Concentration of suspended particles $\left(\right.$ mol. $^{-1}$ )

$605 d$ Typical pore diameter

$606 d_{\text {grain }}$ Diameter ofsand grains

$607 D_{m}$ Diffusion coefficient of particles

$608 \quad D$ Dispersion coefficient

$609 D_{w}$ Self-diffusion coefficient of water

$610 \varepsilon$ porosity

$611 k_{B}$ Boltzmann constant $\left(1.38 \times 10^{-23} \mathrm{~m}^{2} \cdot \mathrm{kg} \cdot \mathrm{s}^{-2} \cdot \mathrm{K}^{-1}\right)$

$612 l_{a d s}$ Typical distance for particles to get adsorbed

$613 Q$ Darcy flux $\left(\mathrm{m}^{-1} \mathrm{~s}^{-1}\right)$

$614 \mu$ Fluid viscosity

$615 R$ Particle relaxivity $\left(\mathrm{mol}^{-1}\right.$. .I. $\left.^{-1}\right)$

$616 r$ Particle radius

$617 \rho$ Surface relaxivity $\left(\mathrm{m} . \mathrm{s}^{-1}\right)$

$618 \rho_{0}$ Surface relaxivity with no adsorbed particles $\left(\mathrm{m} . \mathrm{s}^{-1}\right)$

619 Re Reynolds number

$620 \rho_{w}$ Water density

$621 R_{\text {ads }}$ Pseudo-relaxivity constant of sand surface saturated with adsorbed particles $\left(\mathrm{mol}^{-1} \cdot \mathrm{g} \cdot \mathrm{s}^{-1}\right)$

$622 S$ Concentration of adsorbed particles $\left(\mathrm{mol}^{-\mathrm{g}^{-1}}\right)$

$623 \quad S$ NMR signal

$624 S_{0}$ NMR signal amplitude

$625 \tau$ Detection time

$626 T_{E}$ Echo time

$627 T_{1}$ Longitudinal relaxation time

$628 T_{2}$ Transverse relaxation time

$629 T_{2, \text { bulk }}$ Transverse relaxation time in -particle free- bulk water

$630 T$ Temperature

$631 T_{D}$ Time required for $95 \%$ particles adsorption

$632 \quad V$ Volume area of the pores

$633 v$ Mean velocity through the pores

634

635

5 References 
Adamczyk, Z.: Kinetics of diffusion-controlled adsorption of colloid particles and proteins. J. Colloid Interf. Sci. 229.2, 477-489 (2000)

Baumann, T., Werth, C.J.: Visualization of colloid transport through heterogeneous porous media using magnetic resonance imaging. Colloid. Surface. A 265, 2-10 (2005)

Bear, J.: Dynamics of fluids in porous media. Dover, New York (1988)

Bradford, S. A., Torkzaban, S., Walker, S. L.: Coupling of physical and chemical mechanisms of colloid straining in saturated porous media. Water Res. 41, 3012-3024 (2007)

Bradford, S.A., Yates, S.R., Bettahar, M., Simunek, J.: Physical factors affecting the transport and fate of colloids in saturated porous media. Water Resour. Res. 38(12), 36-1-63-12 (2002)

Brownstein, K. R., Tarr, C. E.: Spin-Lattice relaxation in a system governed by diffusion. J. Magn. Reson. 26, 17-24 (1977)

Bryar, T. R., Daughney, C. J., Knight, R. J.: Paramagnetic effects of iron(III) species on nuclear magnetic relaxation of fluid protons in porous media. J. Magn. Reson. 142, 74-85 (2000)

Callaghan, P.T., Principles of nuclear magnetic resonance microscopy, Clarendon Press, Oxford (1991)

Carr, H., Purcell, E.: Effects of diffusion on free precession in Nuclear Magnetic Resonance Experiments. Phys. Rev. 94, 630 (1954)

Cey, E. E., David, L. R., Passmore, J.: Influence of macroporosity on preferential solute and colloid transport in unsaturated field soils. J. Contam. Hydrol. 107, 45-57 (2009)

Crank, J.: The mathematics of diffusion. Clarendon Press, Oxford (1975)

Diaz, J., Rendueles, M., Diaz, M.: Straining phenomena in bacteria transport through natural porous media. Environ. Sci. Pollut. R. 17, 400-409 (2010)

Dullien, F.A.L.: Porous media - Fluid transport and porous structure. Academic Press, San Diego (1992)

Elimelech, M., Song, L.: Theoretical investigation of colloid separation from dilute aqueous suspensions by oppositely charged granular media. Separ. Technol. 2(1), 2-12 (1992)

Hahn, M. W., Abadzic, D., O'Melia, C. R.: Aquasols: on the role of secondary minima. Environ. Sci. Technol. 38(22), 5915-5924 (2004)

Harding S.G., Baumann H., Nuclear magnetic resonance studies of solvent flow through chromatographic columns: effect of packing density on flow patterns, J. Chromatography A, 905, 1934 (2001)

Israelachvili, J. N.: Intermolecular and surface forces: revised third edition. Academic press (2011) Jacobs, A., Lafolie, F., Herry, J. M., Debroux, M.: Kinetic adhesion of bacterial cells to sand: cell surface properties and adhesion rate. Colloid. Surface. B. 59(1), 35-45 (2007) 
Johnson, P.R., Elimelech, M.: Dynamics of Colloid Deposition in Porous Media: Blocking Based on Random Sequential Adsorption. Langmuir, 11, 801-812 (1995)

Keating, K., Knight, R.: A laboratory study to determine the effect of iron oxides on proton NMR measurements. Geophysics 72, 27-32 (2007)

Keita, E., Faure, P., Rodts, S., Coussot, P.: MRI evidence for a receding-front effect in drying porous media. Phys. Rev. E 87, 062303 (2013)

Ko, C-H., Bhattacharjee, S., Elimelech, M.: Coupled Influence of Colloidal and Hydrodynamic Interactions on the RSA Dynamic Blocking Function for Particle Deposition onto Packed Spherical Collectors. J. Colloid. Interf. Sci. 229, 554-567 (2000)

Korb, J.-P., Monteilhet, L., McDonald, P. J., Mitchell, J.: Microstructure and texture of hydrated cement-based materials: A proton field cycling relaxometry approach. Cement Concrete Res. 37, 295$302(2007)$

Lakshmanan, S., Holmes, W. M., Sloan, W. T., Phoenix, V. R.: Nanoparticle transport in saturated porous medium using magnetic resonance imaging. Chem. Eng. J. 266, 156-162. (2015a)

Lakshmanan, S., Holmes, W. M., Sloan, W. T., Phoenix, V. R.: Characterization of nanoparticle transport through quartz and dolomite gravels by magnetic resonance imaging. Int. J. Environ. Sci. Te. $12,3373-3384(2015 b)$

Lehoux, A. P., Rodts, S., Faure, P., Michel, E., Courtier-Murias, D., Coussot, P.: MRI measurements evidence weak dispersion in homogeneous porous media. Phys. Rev. E 94, 053107 (2016)

Majdalani, S., Michel, E., Di Pietro, L., Angulo-Jaramillo, R., Rousseau, M.: Mobilization and preferential transport of soil particles during infiltration: A core-scale modeling approach. Water Resources Research, 43(5) (2007)

McCarthy, J. F., Zachara, J. M. :Cubsurface transport of contaminants. Environ. Sci. Technol. 23, 496502 (1989)

Meiboom, S., Gill, D.: Modified spin-echo method for measuring nuclear relaxation times. Rev. Sci. Instrum. 29, 688-691 (1958)

Nowach, B., Bucheli, T. D.: Occurrence, behavior and effects of nanoparticles in the environment. Environ. Pollut. 150, 5-22 (2007)

Ochiai, N., Kraft, E. L., Selker, J. S.: Methods for colloid transport visualization in pore networks. Water Resour. Res. 42 (2006)

Ramanan, B., Holmes, W. M., Sloan, W. T., Phoenix, V. R.: Investigation of nanoparticle transport inside coarse-grained geological media using magnetic resonance imaging. Environ. Sci. Technol. 46, 360-366 (2012)

Raychoudhury, T., Tufenkji, N., Ghoshal, S.: Straining of polyelectrolyte-stabilized nanoscale zero valent iron particles during transport through granular porous media. Water Research, 50, 80-89 (2014) 
Ryan, J.N., Elimelech, M.: Colloid mobilization and transport in groundwater. Colloid. Surface. A 107, 1-56 (1995)

Scheven, U.M., Dispersion in non-ideal packed beds. AIChE J. 56.2, 289-297 (2010)

Scheven, U.M., Harris R., and Johns M.L., Intrinsic dispersivity of randomly packed monodisperse spheres, Phys. Rev. Lett. 99, 054502 (2007) Simunek, J., He, C., Pang, L., Bradford, S. A.: Colloid-facilitated solute transport in variably saturated porous media: numerical model and experimental verification. Vadose Zone J., 5 1035-1047 (2006) Song, L., Elimelech, M.: Dynamics of colloid deposition in porous media: modeling the role of retained particles. Colloid. Surface. A 73, 49-63 (1993)

Tosco, T., and Sethi, R.: Transport of non-Newtonian suspensions of highly concentrated micro- and

715 nanoscale iron particles in porous media: A modeling approach. Environmental Science and 716 Technology, 44, 9062-9068 (2010)

717 Tufenkji, N., Elimelech, M.: Breakdown of colloid filtration theory: role of the secondary energy

718 minimum and surface charge heterogeneities. Langmuir 21, 841-852 (2005)

719 Yao, K.M., Habibian, M.T., Omelia, C.R.: Water and waste water filtration. Concepts and applications.

720 Environ. Sci. Technol. 5, 1105-1112 (1971) 